\title{
PROGRESS IN BUILDING OF CESIUM FOUNTAIN FREQUENCY STANDARD AT NPL, INDIA
}

\author{
Amitava Sen Gupta, Ashish Agarwal, Poonam Arora and Kavindra Pant \\ Time and Frequency Section, National Physical Laboratory India, CSIR \\ Dr. K. S. Krishnan Road, New Delhi 110012, India
}

\begin{abstract}
$\underline{\text { Abstract }}$
We describe the current state of progress of the Cesium fountain frequency standard development at the National Physical Laboratory India (NPLI). The optical set-up of the fountain needed to capture, cool, manipulate and detect the cesium atoms is discussed in detail. The concept and design of physical structure of the fountain is also described. In addition, some of the recent results on cooling and trapping of cesium atoms are reported.
\end{abstract}

\section{$\underline{\text { Introduction }}$}

Atomic fountain clocks based on laser cooling and trapping of atoms have been reviewed by Wynands and Weyers [1]. The accuracy level achieved by cesium fountain frequency standard is highest among any other measurement device currently available. Such atomic fountains are being developed at several laboratories around the world [2-3] and a few of them operate as primary frequency standards. At NPLI we have started developing a cesium fountain clock a few years back. The aim of the entire activity is to build a primary frequency standard with a relative uncertainty at about $1 \times 10^{-15}$.

\section{The NPLI Cesium Fountain Clock}

In this paper, the current status of the progress made in building NPLI's primary frequency standard is reported. The concept and design of various parts of the fountain physical structure are discussed in detail.

\section{Optical system}

Generation of atom cooling, repump, launch and detection beams, and finally coupling them onto 8 optical fibers is done on an environmentally controlled vibration free optical table of dimensions $1.04 \mathrm{~m}$ by $1.88 \mathrm{~m}$. An Extended cavity diode laser (ECDL) in Littrow mode is frequency locked to a caesium $\mathrm{D}_{2}$ line [crossover peak of ${ }^{133} \mathrm{Cs} 66^{2} \mathrm{~S}_{1 / 2}(\mathrm{~F}=4)$ $\rightarrow 6^{2} \mathrm{P}_{3 / 2}\left(\mathrm{~F}^{\prime}=4\right.$ and 5$)$ at $\left.852 \mathrm{~nm}\right]$, generated by high resolution saturated absorption spectroscopy using the $110 \mathrm{MHz}$ frequency shifted beam from an acousto-optic modulator (AOM). A tapered amplifier is used to amplify the frequency locked laser beam. For polarization gradient cooling, a double pass
AOM was optimized to ramp down the intensity of the laser within $2 \mathrm{~ms}$ to about $0.5 \mathrm{~mW} \mathrm{~cm}{ }^{-2}$, together with a simultaneous increase of the detuning. Double pass bandwidth of $40 \mathrm{MHz}$ was achieved in order to implement the polarization gradient cooling. This beam was amplified by a Tapered Amplifier system to obtain all the cooling and detection beams required in a fountain frequency standard. More than $400 \mathrm{~mW}$ of frequency locked optical power is generated by this system. The beam was split and directed to three cat's eye double pass arrangements of AOM [2]. The output of first AOM is further split into six separate beams, namely the four horizontal cooling beams $X_{1}$, $\mathrm{X}_{2}, \mathrm{Y}_{1}$ and $\mathrm{Y}_{2}$ (in $\mathrm{x}$ and $\mathrm{y}$ directions) and two detection beams $\left(D_{1}, D_{2}\right)$. The other two AOMs gives two vertical beams $Z_{1}$ and $Z_{2}$. The atoms will be launched by upward directed beam tuned to a frequency $v_{\mathrm{c}}+\delta v$ and the downward-directed beam to $v_{\mathrm{c}}-\delta v$. In addition, home-built, fast mechanical shutters were installed at appropriate places to eliminate residual scattering of resonant light during the Ramsey time.

In Cs, while the cooling laser beams addresses the cyclic transition $6^{2} \mathrm{~S}_{1 / 2} \mathrm{~F}=4 \rightarrow 6^{2} \mathrm{P}_{3 / 2} \mathrm{~F}^{\prime}=5$, nonresonant spontaneous emission populates $6^{2} \mathrm{~S}_{1 / 2} \mathrm{~F}=3$ level eventually. Since this level is not optically coupled to the cooling transition, laser-cooling process comes to a halt in due course. To avoid such a population trapping in $6^{2} \mathrm{~S}_{1 / 2} \mathrm{~F}=3$ level, a second (re-pumping) laser is tuned and locked to the transition ${ }^{133} \mathrm{Cs}^{2} \mathrm{~S}_{1 / 2}\left(\mathrm{~F}=3, \mathrm{M}_{\mathrm{f}}=0\right) \rightarrow 6^{2} \mathrm{P}_{3 / 2}\left(\mathrm{~F}^{\prime}=4\right.$, $\mathrm{M}_{\mathrm{f}}=0$ ). The output beam is split and mixed with one of the cooling beams, $\mathrm{Y}_{2}$, and a detection beam, $\mathrm{D}_{2}$.

Single mode polarization-maintaining optical fibers (PMFs) transfer the 6 cooling and repump beams and 2 detection beams to the Physics Package from the optical table. Transmission efficiency of around 50\% is obtained for all eight fibers. At the output end of the fibers, the beams are collimated with home made beam expanders and give out the desired beam size and polarization. 


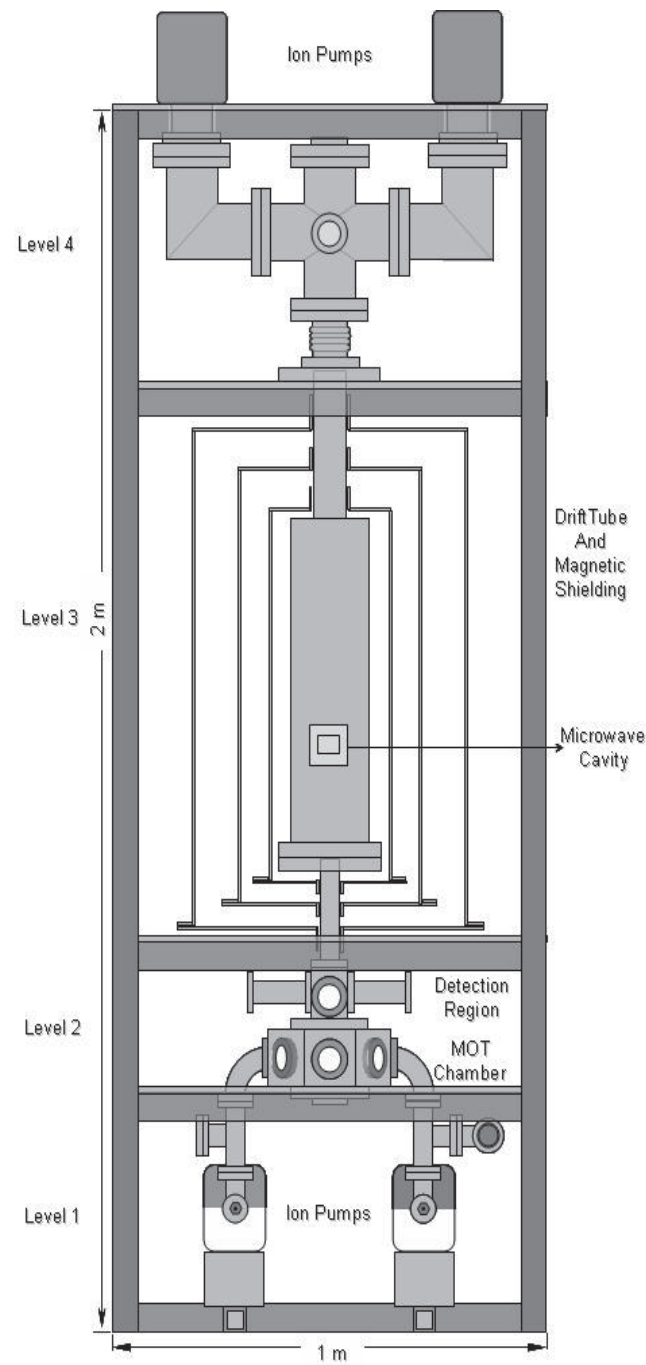

Fig. 1 Schematics of Physics package of the NPLI fountain.

\section{$\underline{\text { Physics Package }}$}

The Physics package of our fountain is shown in Fig. 1. The structure has a base area of 0.85 $\mathrm{m}^{2}$ and is $2 \mathrm{~m}$ tall. As shown in Fig. 1 it is divided into four main levels. Level 1 contains the two 20 /s ion pumps, level 2 contains the magneto-optical trap (MOT) and the fluorescence detection region, level 3 contains the Aluminium drift tube and magnetic shielding and level 4 has the two more $55 \mathrm{l} / \mathrm{s}$ ion pumps. The Cesium atoms are cooled in a MOT followed by Optical Molasses in an octagonal stainless steel chamber with optical viewports. The vacuum enclosures in the atom cooling zone (levels 1 \& 2), drift zone (level 2) and the top zone (level 3) have been individually assembled and tested for vacuum performance. The level of vacuum reached overall is better than $2 \times 10^{-9}$ torr. The source of Cs is a temperature controlled cold finger containing a $\mathrm{Cs}$ ampoule attached to the MOT chamber. The microwave interrogation is proposed to be performed using a TE011 cavity made of OFHC copper inside the drift region. The entire drift region is shielded using three layers of mu metal enclosures with an overall shielding of better than $10^{5}$. A uniform Cfield is produced using a solenoid and compensation coils to give magnetic field homogeneity of about 1 nT, as shown in Fig. 2

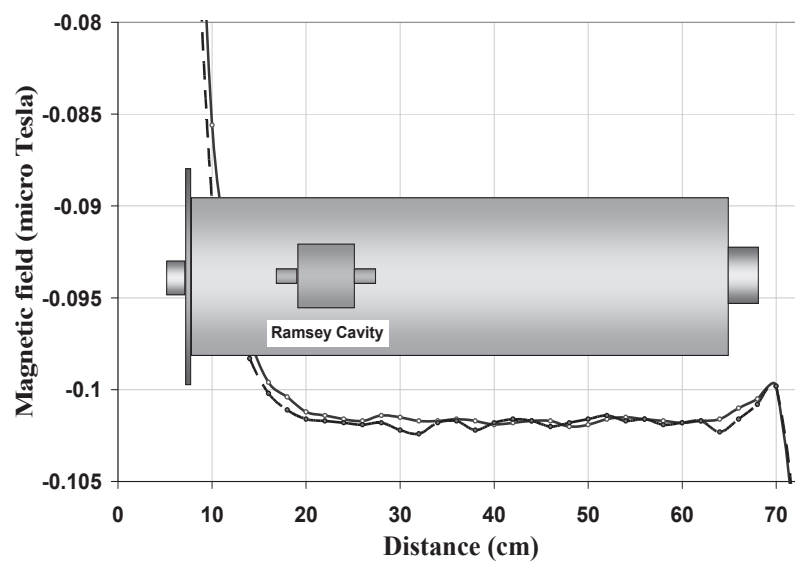

Fig. 2 Experimental study of the $\mathrm{C}$-field variation in the drift region

At present we have succeeded in producing a cold atom cloud of about $10^{7} \mathrm{Cs}$ atoms using the MOT. These are being further cooled using the polarization gradient cooling before being launched.

\section{$\underline{\text { Conclusion }}$}

The status of the NPL Cs fountain at the present time is i) The entire optics is completely assembled and operational, ii) the mechanical subassemblies of the physics package including the vacuum chambers have been put together and individually tested, iii) most of the electronics has been designed and tested and finally iv) a cold atom cloud of about $10^{7} \mathrm{Cs}$ atoms has been produced in a MOT.

\section{References}

[1] R. Wynands and S. Weyers, Metrologia, 42, S64, 2005.

[2] D. M. Meekhof, S. R. Jefferts, M. Stepanovic and T. E. Parker, "Accuracy evaluation of a cesium fountain frequency standard at NIST," IEEE Trans. Instrum. Meas., vol. 50, pp. 507-509, Apr. 2001.

[3] S. Weyers, U. Huebner, R. Schroeder, C. Tamm and A Bauch, "Uncertainty evaluation of the atomic cesium fountain of the PTB," Metrologia, Vol. 38, pp. 343-352, 2001. 